\title{
Patients With cT1N0M0 Oral Squamous Cell Carcinoma Benefit From Elective Neck Dissection: A SEER-Based Study
}

\author{
Alimujiang Wushou, DDS, MD,*; Meng Wang, MD ${ }^{1, *}$; Feiluore Yibulayin, MD2,*; Lei Feng, MD1; \\ Meng-meng Lu, DDS, MDํㅜ Yuan Luo, DDS, MD ${ }^{1}$; Hui Liu, DDS, MD ${ }^{1}$; and Zhi-cheng Yang, DDS, MD ${ }^{1}$
}

\begin{abstract}
Background: The incidence of oral squamous cell carcinoma (OSCC) is increasing, with an estimated 369,000 new patients each year worldwide. Surgery is the primary treatment modality for early-stage OSCC, but there is scant evidence to prove the value of elective neck dissection (END) for relatively small early-stage OSCC. This study aimed to identify factors predicting survival for patients with clinical stage T1NOM0 (cT1NOM0) OSCC and whether up-front END improved survival. Patients and Methods: Patients with cT1N0M0 OSCC who underwent tumor resection with or without END were identified and extracted from the SEER database. Kaplan-Meier survival analysis was used to assess overall survival and diseasespecific survival. Prognostic factors were determined using Cox regression analysis. Results: A total of 5,752 patients with cT1NOMO OSCC were extracted, of whom 2,194 (38.1\%) underwent tumor resection surgery with concurrent END and 3,558 (61.9\%) underwent only tumor resection. In a multivariate Cox analysis, a relatively advanced age ( $>62$ years) and relatively high pathologic grade were the significant negative predictors, but married status (hazard ratio, $0.709 ; P=.006$ ) and undergoing END (hazard ratio, 0.708; $P<.001)$ were identified as significant independent positive factors. Conclusions: Patients with $\mathrm{CT} 1 \mathrm{NOMO}$ OSCC gain significant overall and disease-specific survival benefit from END.
\end{abstract}

J Natl Compr Canc Netw 2021;19(4):385-392 doi: $10.6004 /$ jnccn.2020.7632

\footnotetext{
${ }^{1}$ Department of Oral and Maxillofacial Surgery and Oral Biomedical Engineering Laboratory, Shanghai Stomatological Hospital, Fudan University, and ${ }^{2}$ Department of Preventive Medicine, School of Public Health, Shanghai Medical College, Fudan University, Shanghai, China.

*These authors contributed equally to this study.
}

See page $\mathbf{4 7 4}$ for related commentary.

\section{Background}

Oral squamous cell carcinoma (OSCC) is among the most common cancers worldwide, with approximately 369,000 new cases reported in 2012, and increasing each year. ${ }^{1}$ As presented in the NCCN Clinical Practice Guidelines in Oncology (NCCN Guidelines) for Head and Neck Cancers, ${ }^{2}$ surgery is the primary treatment modality for all stages of OSCC, whereas the addition of adjuvant therapeutic regimens, including radiation therapy and chemotherapy, and the use of targeted therapy are indicated for the most advanced disease (stages III-IV). ${ }^{3-5}$ With regard to the neck management of patients with clinically node-negative early-stage OSCC, there are no consistent conclusions or standardized policies because of the presence of occult neck metastases that are clinically and radiologically undetectable. ${ }^{6-8}$ This practice has especially applied to T1 OSCC tumors $<10 \mathrm{~mm}$ in diameter with no evidence of metastasis in the neck, which have historically been treated with local primary tumor resection only. However, occasionally some of these patients may not be salvageable when untreated occult neck metastases become clinically apparent. ${ }^{9}$ A United Kingdom nationwide study that enrolled 250 randomized and 346 observational cohort patients from 27 hospitals found occult neck disease in $19.1 \%$ and $34.7 \%$ of patients with clinical stage $\mathrm{T} 1$ and $\mathrm{T} 2$ disease, respectively. ${ }^{10}$

The NCCN Guidelines suggest that patients with initial preoperative staging of at least levels I-III may undergo elective neck dissection (END), which is determined at the discretion of the surgeon ${ }^{2}$ But it is still debatable whether patients with early-stage nodenegative OSCC should receive END. Many clinicians in head and neck surgery still follow the protocol of performing selective techniques on patients with clinically negative necks (N0), but only when the tumor size exceeds $\geq 4 \mathrm{~cm}$ (T2). END requires a longer, more complex operation than tumor resection alone and increases the complication risk. In considering the necessity of END versus the benefit, some surgeons choose not to offer up-front END due to concern about overtreatment, reserving neck dissection as a salvage treatment for 
subsequent neck metastasis. Similarly, some studies have concluded that patients with node-negative OSCC should not receive up-front END to avoid additional complications and costs. ${ }^{11,12}$ Currently, increasingly more researchers advocate that END should be performed routinely during resection of the primary tumor, in consideration of postoperative recurrence and poor prognosis due to the high rate of occult cervical metastasis in early OSCC. ${ }^{13}$

Because of the lack of consensus in characteristics, study populations, and patient management, the results supporting either recommendation may not be readily generalizable. ${ }^{14-16}$ However, although a meta-analysis from $2011^{17}$ and prospective randomized studies from $2015^{18}$ and $2019^{10}$ showed a benefit for END in both T1N0 and T2N0 OSCC, 2 retrospective studies ${ }^{13,19}$ and the UK prospective randomized study and meta-analysis ${ }^{10}$ have shown a benefit for T1N0 OSCC alone. The difference between the management of T1N0 and T2N0 is still a contentious point.

To our knowledge, only one article to date has reported a prospective randomized study and metaanalysis that quantifies the benefit of END in T1N0 and T2N0 OSCC. ${ }^{10}$ Our study concentrated on patients with only clinical stage T1N0M0 (cT1N0M0) OSCC. We aimed to gather sufficient evidence to make strong, wellsupported recommendations to either support or refute the findings of the single published study, which suggested that patients with cT1N0M0 OSCC gained significant overall survival (OS) and disease-specific survival (DSS) benefit. ${ }^{10}$ In addition, we sought to determine the clinical features and prognostic factors associated with cT1NOM0 OSCC that affected OS and DSS in this patient cohort. To address these issues, a retrospective investigation was conducted using a study population from the internationally renowned SEER database.

\section{Patients and Methods}

The study population was extracted from the SEER database using its exclusive software (SEER*Stat 8.3.6). Patients were identified via ICD-O-3 as described elsewhere. $^{20}$ Briefly, patients with OSCC were selected through the ICD-O-3 morphologic and topographic codes 8050-8076, 8078, 8083, 8084, 8094, C01.9, C02.0, C02.1, C02.2, C02.3, C02.8, C02.9, C03.0, C03.1, C03.9, C04.0, C04.9, C05.0, C06.0, C06.1, and C06.2. We then selected only patients with primary AJCC stage I OSCC. Because our study used established data and did not involve interactions with human patients, Institutional Review Board approval was not required.

\section{Statistical Analysis}

Clinical and demographic features were compared across subgroups using the chi-square or Fisher exact test.
Survival curves were generated using the Kaplan-Meier method, and the log-rank test was used to evaluate survival differences between subgroups. Adjusted hazard ratios (HRs) along with 95\% confidence intervals were calculated using univariate and multivariate Cox proportional hazards regression models. Statistical analysis was performed using the statistical packages $\mathrm{R}$ version 3.4.3 (R Foundation for Statistical Computing), Empower $\mathrm{R}$ (X \& Y Solutions Inc.), and SPSS Statistics, version 23 (IBM Corp). $P$ values $<.05$ were considered statistically significant, and all statistical tests were 2-tailed.

\section{Results}

\section{Summary Statistics}

In total, 5,752 patients with cT1N0M0 OSCC who fulfilled all inclusion criteria were identified: 3,209 (55.8\%) were male, and 2,543 (44.2\%) were female. The follow-up time period for the cohort ranged from 1 to 155 months, with a mean follow-up of 62.43 months (SD, 39.90). Mean patient age at diagnosis was 62.02 years (SD, 13.74; range, 21-105 years). Furthermore, nearly $55 \%$ of all patients in this cohort were from the age groups of 50-59 and $60-69$ years. Most patients were White $(n=5,004 ; 87.9 \%)$, followed by other races $(n=511 ; 9.0 \%)$ and Black $(n=176$; $3.1 \%)$. Most patients were married ( $\mathrm{n}=3,260 ; 62.0 \%)$, but $831(15.8 \%)$ were single and 1,167 (22.2\%) were listed as other. The numbers of patients with histologic grade 1,2 , or $3 / 4$ disease were 1,949 (37.6\%), 2,757 (53.1\%), and 483 (9.3\%), respectively.

In this cohort, 2,194 patients $(38.1 \%)$ underwent END concurrent with tumor resection, whereas 3,558 (61.9\%) received only tumor resection with a wait-andobserve approach for the neck.

In addition, there were 4,778 patients with OSCCspecific mortality data available for DSS analysis, including 2,154 women (45.1\%) and 2624 men (54.9\%). Mean age was 60.46 years (SD, 13.38; range, 21-105 years), and mean follow-up time was 64.22 months (range, 1-155 months). Basic clinical and demographic characteristics are summarized in Table 1, and notable differences were detected between subgroups.

\section{Survival Analysis}

Survival analysis was performed using Kaplan-Meier estimate for OS. There were significant differences in OS depending on age group $(P<.0001)$, age at diagnosis $(P<.0001)$, sex $(P=.0026)$, marital status at diagnosis $(P<.0001)$, race $(P<.0001)$, primary tumor site $(P<.0001)$, pathologic grade $(P<.0001)$, and neck dissection status $(P=.0004)$ (Figure 1). DSS analysis was performed the same as OS analysis, and significant differences were found for age group $(P<.0001)$, age at diagnosis $(P<.0001)$, pathologic grade $(P<.0001)$, tumor site $(P=.0002)$, marital 


\begin{tabular}{|c|c|c|c|c|c|c|c|c|c|c|c|c|c|c|c|c|}
\hline \multirow[b]{3}{*}{ Variable } & \multicolumn{8}{|c|}{ Disease-Specific Survival } & \multicolumn{8}{|c|}{ Overall Survival } \\
\hline & \multicolumn{4}{|c|}{ Wait and Observe } & \multicolumn{4}{|c|}{ END } & \multicolumn{4}{|c|}{ Wait and Observe } & \multicolumn{4}{|c|}{ END } \\
\hline & Alive & Dead & Total & $\begin{array}{c}P \\
\text { Value }\end{array}$ & Alive & Dead & Total & $\begin{array}{c}P \\
\text { Value }\end{array}$ & Alive & Dead & Total & $\begin{array}{c}P \\
\text { Value }\end{array}$ & Alive & Dead & Total & $\begin{array}{c}P \\
\text { Value }\end{array}$ \\
\hline Sex & & & & .997 & & & & .036 & & & & .041 & & & & .001 \\
\hline Male & 1,331 & 230 & 1,561 & & 925 & 138 & 1,063 & & 1,362 & 572 & 1,934 & & 937 & 338 & 1,275 & \\
\hline Age range, $y$ & & & & $<.001$ & & & & $<.001$ & & & & $<.001$ & & & & $<.001$ \\
\hline $20-29$ & 30 & 1 & 31 & & 33 & 1 & 34 & & 30 & 3 & 33 & & 33 & 2 & 35 & \\
\hline $30-39$ & 105 & 5 & 110 & & 114 & 7 & 121 & & 105 & 8 & 113 & & 114 & 10 & 124 & \\
\hline $40-49$ & 311 & 36 & 347 & & 262 & 21 & 283 & & 315 & 55 & 370 & & 264 & 41 & 305 & \\
\hline$\geq 80$ & 204 & 102 & 306 & & 85 & 26 & 111 & & 212 & 295 & 507 & & 86 & 79 & 165 & \\
\hline Race & & & & .247 & & & & .164 & & & & $<.001$ & & & & .001 \\
\hline White & 2,127 & 376 & 2,503 & & 1,421 & 194 & 1,615 & & 2,193 & 903 & 3,096 & & 1,441 & 467 & 1,908 & \\
\hline Black & 69 & 17 & 86 & & 41 & 8 & 49 & & 72 & 39 & 111 & & 41 & 24 & 65 & \\
\hline Other & 243 & 35 & 278 & & 172 & 15 & 187 & & 247 & 58 & 305 & & 173 & 33 & 206 & \\
\hline Marital status & & & & $<.001$ & & & & $<.001$ & & & & $<.001$ & & & & $<.001$ \\
\hline Single & 358 & 56 & 414 & & 252 & 34 & 286 & & 372 & 125 & 497 & & 257 & 77 & 334 & \\
\hline Married & 1,460 & 217 & 1,677 & & 998 & 103 & 1,101 & & 1,495 & 500 & 1,995 & & 1,012 & 253 & 1,265 & \\
\hline Other & 414 & 129 & 543 & & 267 & 61 & 328 & & 430 & 308 & 738 & & 269 & 160 & 429 & \\
\hline Lower gum & 101 & 19 & 120 & & 70 & 13 & 83 & & 106 & 47 & 153 & & 71 & 32 & 103 & \\
\hline Hard palate & 49 & 12 & 61 & & 6 & 0 & 6 & & 52 & 22 & 74 & & 6 & 0 & 6 & \\
\hline $\begin{array}{l}\text { Cheek mucosa \& } \\
\text { vestibule of } \\
\text { mouth }\end{array}$ & 130 & 38 & 168 & & 78 & 9 & 87 & & 134 & 69 & 203 & & 78 & 18 & 96 & \\
\hline Retromolar area & 55 & 15 & 70 & & 40 & 10 & 50 & & 59 & 33 & 92 & & 43 & 29 & 72 & \\
\hline Tongue & 1,735 & 271 & 2,006 & & 1,186 & 128 & 1,314 & & 1,779 & 611 & 2,390 & & 1,199 & 296 & 1,495 & \\
\hline Floor of mouth & 309 & 57 & 366 & & 246 & 57 & 303 & & 321 & 176 & 497 & & 250 & 147 & 397 & \\
\hline
\end{tabular}

Abbreviations: END, elective neck dissection; OSCC, oral squamous cell carcinoma.

status at diagnosis $(P<.0001)$, and neck dissection status $(P=.0037)$ (Figure 2$)$.

\section{Multivariate Analysis}

To determine the efficacy of END based on various clinicopathologic characteristics, all factors identified as significant in the survival analysis were entered into a multivariate analysis based on the Cox regression model. Details of the multivariate Cox regression analysis are presented in Figure 3. In addition to age, pathologic differentiation of OSCC was an important independent prognostic indicator. A relatively high pathologic grade (grade 2 or $3 / 4$ ) was adversely associated with DSS (grade 2: HR, 2.019; 95\% CI, 1.650-2.470; $P=.000$; grade 3/4: HR, 3.078; 95\% CI, 2.340-4.048; $P<.0001$ ) and OS (grade 2: HR, 1.450; 95\% CI, 1.280-1.644; $P=.000$; grade 3/4: HR, 1.826; 95\% CI, 1.154-2.203; $P=.000)$ compared with grade 1 . More important, END was favorably associated with 
A

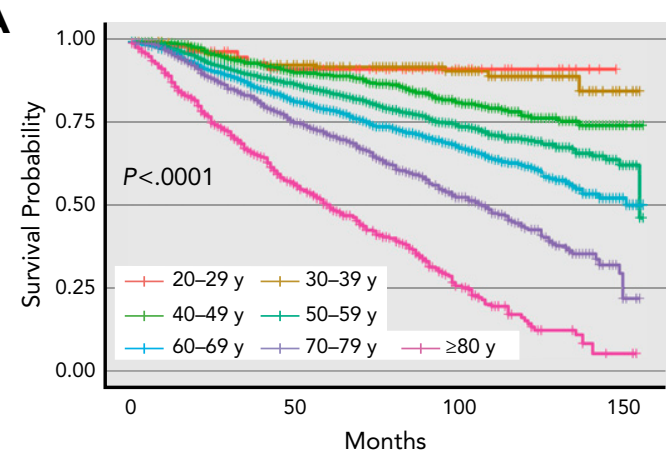

C

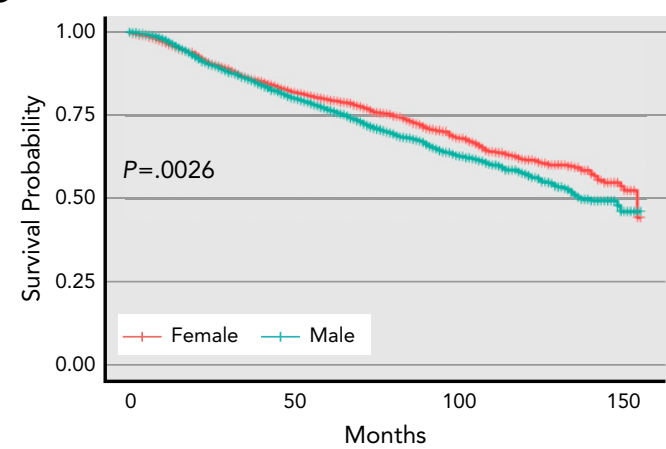

E

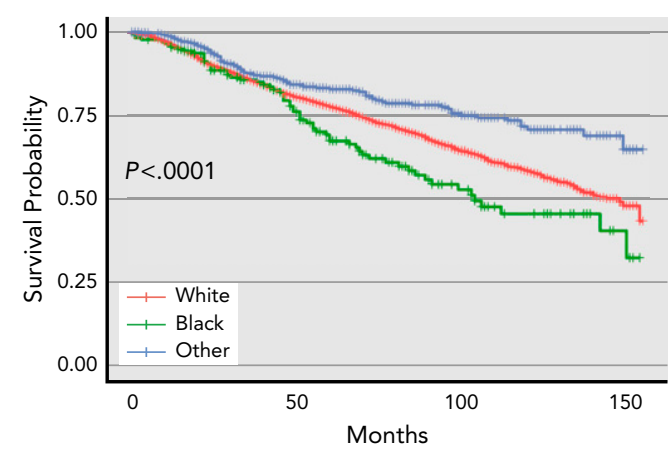

G

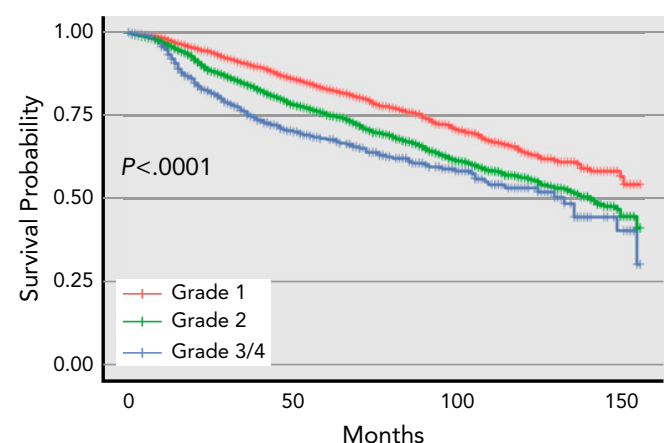

B

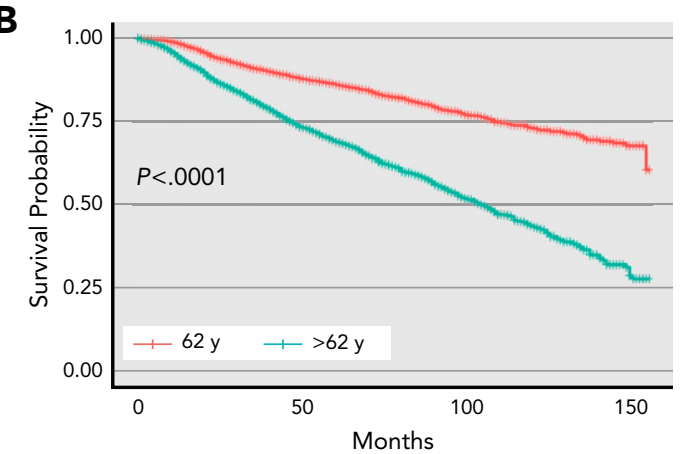

D

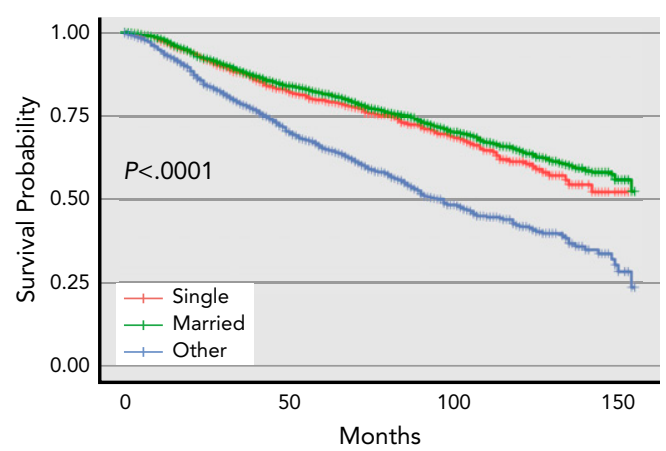

F

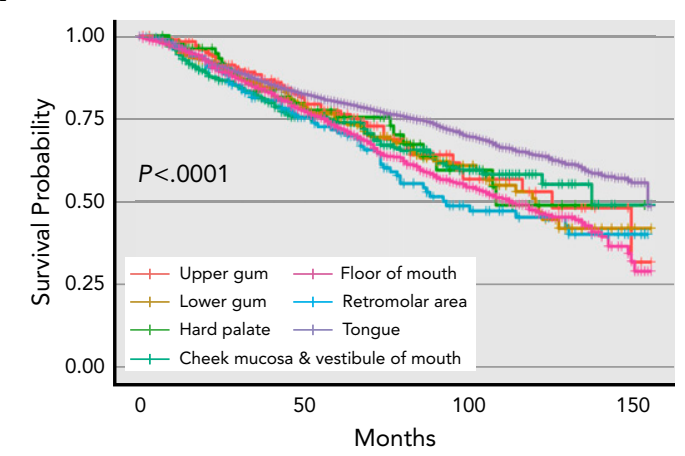

H

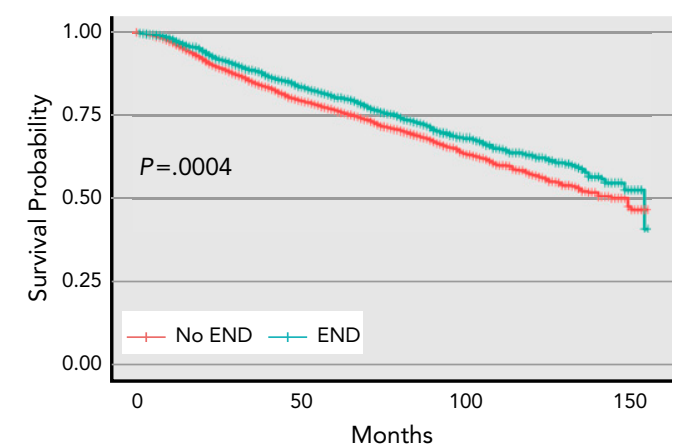

Figure 1. Overall survival curves of patients with cT1NOMO oral squamous cell carcinoma compared according to (A) age range, (B) mean age at diagnosis (62 years), (C) sex, (D) marital status at diagnosis, (E) race, (F) tumor orientation, (G) pathologic differentiation, and (H) END. Abbreviations: cT1NOMO, clinical stage T1NOMO; END, elective neck dissection. 
A

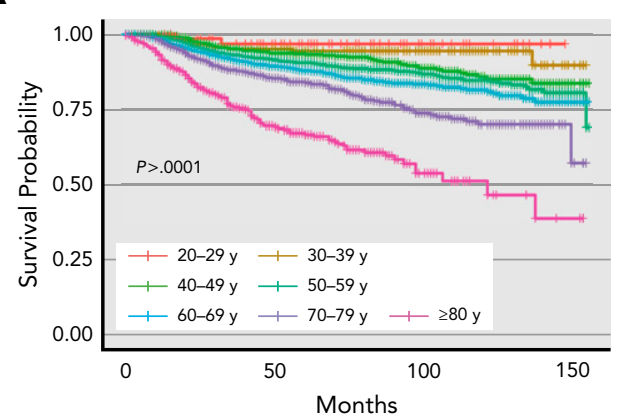

\begin{tabular}{lrrrr}
\multicolumn{2}{l}{ Number at risk } & & & \\
$20-29 y$ & 65 & 42 & 16 & 0 \\
$30-39 y$ & 231 & 154 & 68 & 6 \\
$40-49 y$ & 630 & 425 & 200 & 19 \\
$50-59$ y & 1,345 & 812 & 361 & 24 \\
$60-69 y$ & 1,318 & 720 & 272 & 22 \\
$70-79 y$ & 772 & 385 & 126 & 7 \\
$\geq 80 y$ & 417 & 154 & 30 & 3
\end{tabular}

\section{C}

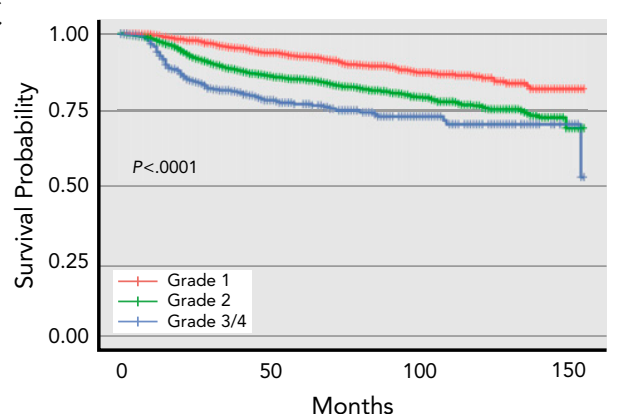

Number at risk

$\begin{array}{lr}\text { Grade } 1 & 1,659 \\ \text { Grade } 2 & 2,260 \\ \text { Grade 3/4 } & 395\end{array}$
B

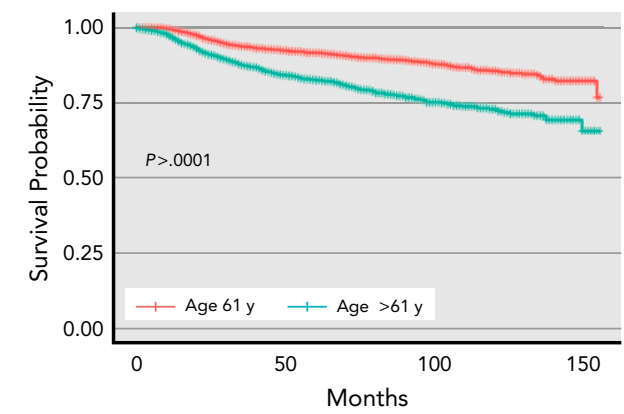

Number at risk

$\begin{array}{lllll}\text { Age } 61 \mathrm{y} & 2,432 & 1,531 & 683 & 50 \\ \text { Age }>61 \mathrm{y} & 2,346 & 1,161 & 390\end{array}$

D



Number at risk

Upper gum $\quad 144$

Lower gum 203

Hard palate $\quad 67$

Cheek mucosa \& 255

vestibule of mouth

Retromolar area 120

Tongue $\quad 3,320$

Floor of mouth $\quad 669$

\section{$\mathbf{F}$}

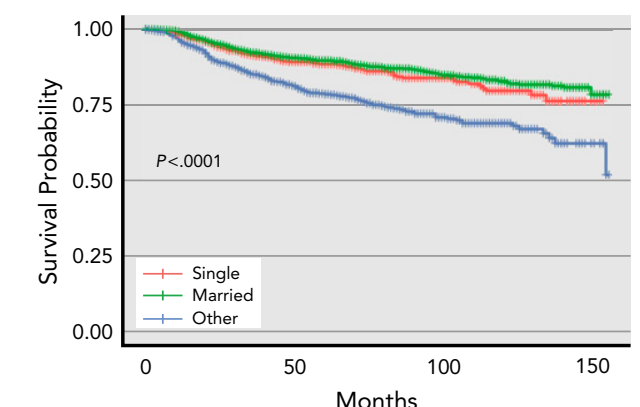

Number at risk

$\begin{array}{lrrrr}\text { Single } & 700 & 399 & 154 & 7 \\ \text { Married } & 2,778 & 1,633 & 677 & 55 \\ \text { Other } & 871 & 424 & 160 & 13\end{array}$

Figure 2. Disease-specific survival curves of patients with $\mathrm{cT1N0M0}$ oral squamous cell carcinoma compared according to (A) age range, (B) mean age at diagnosis (61 years), (C) pathologic differentiation, (D) tumor orientation, (E) marital status at diagnosis, and (F) END. Abbreviations: cT1NOM0, clinical stage T1NOM0; END, elective neck dissection. 
A

\begin{tabular}{|c|c|c|c|}
\hline Variable & HR & HR $(95 \% \mathrm{CI})$ & $P$ Value \\
\hline Cheek \& vestibule vs upper gum & $=$ & $2.282(1.281-4.063)$ & .005 \\
\hline Marital status: married vs single & 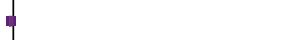 & $0.709(0.554-0.908)$ & .006 \\
\hline Age group: $60-69$ vs $20-29$ y & $\longmapsto$ & $4.137(1.017-16.834)$ & .047 \\
\hline Age group: $70-79$ vs $20-29$ y & $\longmapsto$ & $5.702(1.397-23.269)$ & .015 \\
\hline Age group: $\geq 80$ vs $20-29$ y & $\longrightarrow$ & $10.826(2.638-44.424)$ & .001 \\
\hline Pathologic grade: 2 vs 1 & 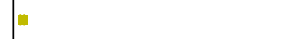 & $2.019(1.650-2.470)$ & .000 \\
\hline Pathologic grade: $3 / 4$ vs 1 & ! & $3.078(2.340-4.048)$ & .000 \\
\hline \multirow[t]{2}{*}{ Neck dissection: yes vs no } & & $0.708(0.592-0.846)$ & .000 \\
\hline & 30 & & \\
\hline
\end{tabular}

B

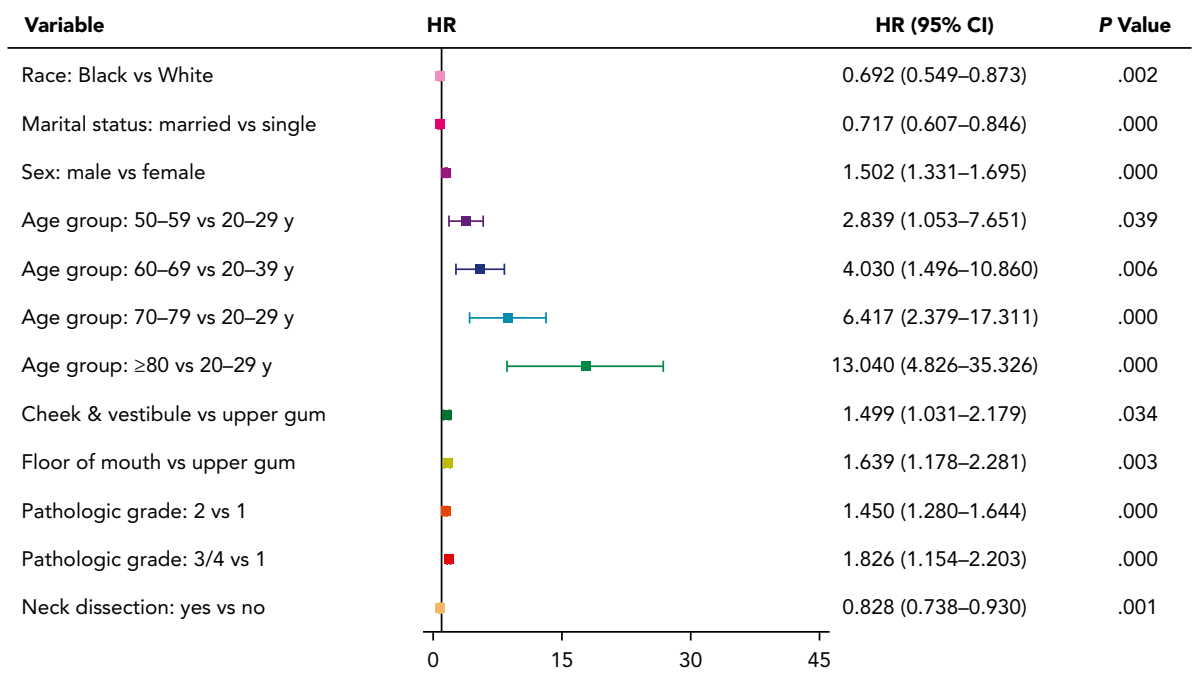

Figure 3. Forest plots summarizing HR for (A) disease-specific survival and (B) overall survival.

Abbreviation: $H R$, hazard ratio.

prolonged DSS (HR, 0.708; 95\% CI, 0.592-0.846; $P=.000$ ) and OS (HR, 0.828; 95\% CI, 0.738-0.930; $P=.001$ ) compared with observation.

\section{Discussion}

Previously, age and sex were reportedly not associated with OSCC survival rate. In contrast, in our survival analyses depending on demographic factors such as age, sex, and race and concentrating on only cT1NOM0 OSCC, all of these factors were independent prognostic indicators for OS. Furthermore, our results showed a lower DSS rate among elderly individuals (aged $>61$ years) compared with younger individuals, which was consistent with the findings of some previous studies. ${ }^{21,22}$ With regard to marital status, our results showed that married status at diagnosis had a significant independent protective effect on cT1N0M0 OSCC survival. Another study that aimed to evaluate the impact of marital status on OSCC survival supported the same conclusion. ${ }^{23}$
However, more research is needed on the association of marital status with increased survival.

To date, many studies have confirmed that histologic grade correlates with survival rate in patients with OSCC. ${ }^{19,24}$ Our study produced a similar conclusion. Typical survival differences were found among different pathologic grades for both DSS and OS. Based on our retrospective analysis of a large cohort of 5,752 patients, we confirmed that tumor site is another important factor affecting patient outcome. Significantly poorer DSS and OS were observed for tumors originating from the cheek mucosa and vestibule of the mouth compared with the upper gum. We tentatively interpret this as a probable consequence of the increased incidence of cervical lymph node metastasis and the relatively complicated resection procedure for tumors in the cheek mucosa and vestibule of the mouth. Similarly, OSCC site played an important role in disease prognosis, as presented in another study. ${ }^{25}$ 
One of the most common causes of treatment failure in early-stage OSCC is the high incidence of occult metastases and recurrence in the neck. It has also been suggested that recurrence in the neck is associated with a significant decrease in DSS. ${ }^{26}$ A prospective randomized controlled trial of 496 patients showed that those who received END at the time of the primary surgery were less likely to experience nodal recurrence $(29.6 \%)$ than those who did not $(45.1 \%) .{ }^{18}$ In a retrospective administrative database analysis, END was proven to improve DSS versus neck observation in patients with T1/T2N0M0 OSCC of the tongue with moderately differentiated, poorly differentiated, or undifferentiated histologic grade tumors $>1 \mathrm{~cm}$. However, those with well-differentiated tumors benefited from END only when the tumor was $>2 \mathrm{~cm} .{ }^{13}$ Meanwhile, a recent study also recommended END for moderately and poorly differentiated T1N0 tumors irrespective of depth of invasion but did not support the idea that END may be necessary for well-differentiated tumors. ${ }^{19}$ In another prospective randomized study and meta-analysis of 6 randomized trials of END in early-stage OSCC, ${ }^{10}$ the pooled HR for OS indicated a $31 \%$ reduction in the risk of death with END (HR, 0.69; $P=.002$ ) and a $33 \%$ reduction in the risk of recurrence/death (HR, $0.67 ; P=.04$ for DSS). It also showed a benefit for T1 tumors and even for tumors $<10 \mathrm{~mm}$ in diameter.

However, the 2009 prospective randomized study by Yuen et $\mathrm{al}^{8}$ showed no benefit for END in patients with OSCC of the tongue, with a 5-year DSS rate of $87 \%$ for the observation arm and $89 \%$ for the END arm (the $2 \%$ difference was not significant). In a study on variation in OSCC management strategies between representatives of head and neck cancer treatment centers, high variation in management of the neck was seen in early OSCC. ${ }^{6}$ There appears to be a need for direct evidence on optimal management decisions to establish more evidence-based management and uniform practice. Many surgeons still underestimate the rate at which occult neck metastases occur in patients with smaller oral tumors, especially $\mathrm{T} 1$ tumors.

We performed this analysis to determine the effect of END for patients with only cT1N0M0 OSCC. Our findings showed that END significantly improved both OS and DSS in these patients, which is consistent with the findings of 2 large-scale prospective randomized studies and a meta-analysis of all prospective randomized studies. ${ }^{10,18}$ Our retrospective analysis using the SEER database to analyze 5,752 patients with only cT1N0M0 OSCC complements those studies, and together with the meta-analysis provides evidence supporting a review of head and neck cancer guidelines.

However, several patient characteristics in the 2 previous studies differed from ours: (1) mean ages were 48 years (Mumbai) ${ }^{18}$ and 63 years (UK) ${ }^{10}$ versus
62.02 years in our study, so age was similar between our analysis and the UK trial; (2) patients were 75\% (Mumbai) and $64 \%$ (UK) male versus $55.8 \%$ in our study; (3) $85.6 \%$ of patients in the previous trials were White versus $87.9 \%$ in our study; (4) most patients had well-/moderately differentiated disease (grade 1/2), accounting for $82.3 \%$ (Mumbai) and $70.8 \%$ (UK) of patients in the previous studies versus $90.7 \%$ in our study; and (5) the tongue was the most common site for primary tumors in all 3 studies, with incidence rates of 85\% (Mumbai) and 61\% (UK) versus $67.5 \%$ in our study.

Despite these differences, all 3 studies showed improvements in OS and DSS. Compared with results generated by combining the UK and Mumbai studies, ${ }^{10,18}$ which had a pooled HR for OS of 0.66 (95\% CI, 0.49-0.89; $P=.006)$ and an HR for DSS of 0.53 (95\% CI, 0.37-0.77; $P<.001$ ), our results were more conservative in regard to the benefit of END for OS (HR, 0.828) and DSS (HR, 0.708). In addition, the OS HRs were 0.75 and 0.42 for $\mathrm{T} 1$ and T2 tumors, respectively, in the Mumbai study ${ }^{18}$ (suggesting that END may be a more favorable approach in larger vs smaller tumors), but the difference was not statistically significant. However, evidence from the UK randomized trial ${ }^{10}$ indicated that END also benefits $\mathrm{T} 1$ tumors. Similarly, our study also provides evidence for the benefit derived from END in patients with T1 OSCC.

END is occasionally performed at the same time as tumor resection for cT1N0M0 OSCC. ${ }^{17}$ In terms of postoperative morbidity of the neck and costs related to the procedure, END is associated with an increased risk of adverse effects, including those involving swallowing function and sensory nerves, but has minimal impacts on most quality-of-life components. ${ }^{10}$ One study presented a cost-effectiveness analysis of END in the initial surgical management of early-stage oral cavity cancer. ${ }^{27}$ The researchers constructed a Markov model to simulate primary, adjuvant, and salvage therapy; disease recurrence; and survival in patients with $\mathrm{T} 1 / \mathrm{T} 2$ clinically node-negative oral cavity squamous cell carcinoma. Their base-case model found that over a lifetime, the addition of END to primary surgery reduced overall treatment costs by $\$ 6,000$ and improved effectiveness by 0.42 qualityadjusted life-years compared with primary surgery alone. The decrease in overall cost despite the added neck dissection was a result of less use of salvage therapy. Therefore, increasingly more clinicians believe that the addition of END reduces costs and improves health outcomes, making it a cost-effective treatment strategy for patients.

Some limitations of our study should be noted. First, other oncologic outcome data were absent, such as tumor depth of invasion, which has drawn much recent attention as a better predictor of disease prognostics, as represented in the 8th edition of the AJCC Cancer Staging Manual. ${ }^{3}$ The true oncologic benefit 
of END could not be determined accurately. Further research on tumor depth of invasion should be explored. Second, a research data bias may potentially exist for the selection of END due to surgeon bias and patient choice. Because younger patients were more likely to undergo END than older patients, and multiple studies have shown improved OS in younger patients, age may play a role in the frequency of END. Therefore, additional translational studies to identify favorable subgroups of patients should be performed. Third, the long-term complications of neck dissection are not addressed in our study but should be further presented to provide more comprehensive evidence. Finally, information on neck lymph node metastasis after surgery is not included in this analysis but may be used to estimate a more convincing result.

\section{Conclusions}

Older age ( $>62$ years), male sex, high pathologic grade, and unmarried status were associated with an increased risk of death in patients with cT1N0M0 OSCC. END performed at the same time as treatment of the primary site for patients with cT1N0M0 OSCC confers a significant OS and DSS benefit.

\section{Acknowledgments}

We are grateful to American Journal Experts (https://www.aje. com, Durham, NC) for checking the English of the article.

Submitted April 19, 2020; accepted for publication July 30, 2020 Published online January 29, 2021.

Author contributions: Initial research concept: Wang. Study design: Wang Wushou. Lead investigators for data collection: Wushou, Yibulayin. Data analysis: Wang, Yibulayin, Feng. Spreadsheet data management: Lu, Luo. Clerical support: Liu. Guidance on study revision: Yang. Manuscript preparation and revision for important intellectual content: All authors.

Disclosures: The authors have disclosed that they have not received any financial consideration from any person or organization to support the preparation, analysis, results, or discussion of this article.

Correspondence: Zhi-cheng Yang, DDS, MD, Department of Oral and Maxillofacial Surgery, Shanghai Stomatological Hospital, Fudan University, 356 Beijing East Road, Shanghai 200001, China.

Email: zhicheng_yang1998@fudan.edu.cn

\section{References}

1. Ghantous Y, Abu Elnaaj I. [Global incidence and risk factors of oral cancer] Harefuah 2017;156:645-649 [in Hebrew]

2. Pfister DG, Spencer S, Adelstein D, et al. NCCN Clinical Practice Guidelines in Oncology: Head and Neck Cancers. Version 2.2020. Accessed August 5, 2020. To view the most recent version, visit NCCN.org

3. Amin MB, Edge S, Greene F, et al., eds. AJCC Cancer Staging Manual, 8th ed. Basel, Switzerland: Springer International Publishing; 2017.

4. Shah JP, Gil Z. Current concepts in management of oral cancer-surgery. Oral Oncol 2009:45:394-401.

5. Rampino M, Bacigalupo A, Russi E, et al. Efficacy and feasibility of induction chemotherapy and radiotherapy plus cetuximab in head and neck cancer. Anticancer Res 2012;32:195-199.

6. Govers TM, de Kort TB, Merkx MA, et al. An international comparison of the management of the neck in early oral squamous cell carcinoma in the Netherlands, UK, and USA. J Craniomaxillofac Surg 2016;44:62-69.

7. Gill A, Givi B, Moore MG. AHNS series - do you know your guidelines? Assessment and management of malnutrition in patients with head and neck cancer: review of the NCCN Clinical Practice Guidelines in Oncology (NCCN Guidelines). Head Neck 2019;41:577-583.

8. Yuen $\mathrm{AP}, \mathrm{Ho} \mathrm{CM}, \mathrm{Chow} \mathrm{TL}$, et al. Prospective randomized study of selective neck dissection versus observation for NO neck of early tongue carcinoma. Head Neck 2009;31:765-772

9. Tsang RK, Chung JC, To VS, et al. Efficacy of salvage neck dissection for isolated nodal recurrences in early carcinoma of oral tongue with watchful waiting management of initial NO neck. Head Neck 2011;33:1482-1485.

10. Hutchison IL, Ridout F, Cheung SMY, et al. Nationwide randomised trial evaluating elective neck dissection for early stage oral cancer (SEND study) with meta-analysis and concurrent real-world cohort. $\mathrm{Br}$ J Cancer 2019;121:827-836.

11. D'Cruz AK, Siddachari RC, Walvekar RR, et al. Elective neck dissection for the management of the $\mathrm{NO}$ neck in early cancer of the oral tongue: need for a randomized controlled trial. Head Neck 2009;31:618-624.

12. Kelner N, Vartanian JG, Pinto CA, et al. Does elective neck dissection in T1/T2 carcinoma of the oral tongue and floor of the mouth influence recurrence and survival rates? Br J Oral Maxillofac Surg 2014;52:590-597.

13. Patel TD, Vázquez A, Marchiano E, et al. Efficacy of elective neck dissection in T1/T2N0M0 oral tongue squamous cell carcinoma: a population-based analysis. Otolaryngol Head Neck Surg 2016;155: 588-597.

14. Peng KA, Chu AC, Lai C, et al. Is there a role for neck dissection in T1 oral tongue squamous cell carcinoma? The UCLA experience. Am J Otolaryngol 2014;35:741-746.
15. Liu X, Lao X, Liang $L$, et al. Neck observation versus elective neck dissection in management of clinical T1/2N0 oral squamous cell carcinoma: a retrospective study of 232 patients. Chin J Cancer Res 2017;29:179-188.

16. Obayemi A Jr, Cracchiolo JR, Migliacci JC, et al. Elective neck dissection (END) and cNO hard palate and upper gingival cancers: a National Cancer Database analysis of factors predictive of END and impact on survival. J Surg Oncol 2019;120:1259-1265

17. Fasunla AJ, Greene BH, Timmesfeld N, et al. A meta-analysis of the randomized controlled trials on elective neck dissection versus therapeutic neck dissection in oral cavity cancers with clinically node-negative neck. Oral Oncol 2011;47:320-324.

18. D'Cruz AK, Vaish R, Kapre N, et al. Elective versus therapeutic neck dissection in node-negative oral cancer. N Engl J Med 2015;373:521-529.

19. Zhan KY, Morgan PF, Neskey DM, et al. Preoperative predictors of occult nodal disease in cT1 N0 oral cavity squamous cell carcinoma: review of 2623 cases. Head Neck 2018;40:1967-1976.

20. Fakhry C, Krapcho M, Eisele DW, et al. Head and neck squamous cell cancers in the United States are rare and the risk now is higher among white individuals compared with black individuals. Cancer 2018;124: 2125-2133

21. Karadaghy OA, Shew M, New J, et al. Development and assessment of a machine learning model to help predict survival among patients with oral squamous cell carcinoma. JAMA Otolaryngol Head Neck Surg 2019; $145: 1115$

22. Xu Q, Wang C, Li B, et al. The impact of age on oral squamous cell carcinoma: a longitudinal cohort study of 2,782 patients. Oral Dis 2019 i 25:730-741.

23. Shi X, Zhang TT, Hu WP, et al. Marital status and survival of patients with ora cavity squamous cell carcinoma: a population-based study. Oncotarget 2017;8:28526-28543.

24. Kademani D, Bell RB, Bagheri S, et al. Prognostic factors in intraoral squamous cell carcinoma: the influence of histologic grade. J Oral Maxillofac Surg 2005;63:1599-1605

25. Jehn P, Dittmann J, Zimmerer R, et al. Survival rates according to tumour location in patients with surgically treated oral and oropharyngeal squamous cell carcinoma. Anticancer Res 2019;39:2527-2533.

26. Ganly I, Goldstein D, Carlson DL, et al. Long-term regional control and survival in patients with "low-risk," early stage oral tongue cancer managed by partial glossectomy and neck dissection without postoperative radiation: the importance of tumor thickness. Cancer 2013;119:1168-1176.

27. Acevedo JR, Fero KE, Wilson B, et al. Cost-effectiveness analysis of elective neck dissection in patients with clinically node-negative oral cavity cancer. J Clin Oncol 2016;34:3886-3891. 